\title{
HARDNESS RESULTS FOR TOTAL RAINBOW CONNECTION OF GRAPHS
}

\author{
LILY CHEN \\ School of Mathematics Science \\ Huaqiao University \\ Quanzhou 362021, China \\ e-mail: lily60612@126.com \\ BOFENG HuO \\ Department of Mathematics \\ Qinghai Normal University \\ Xining, 810008, China \\ e-mail: hbf@qhnu.edu.cn \\ AND \\ YINGBIN MA \\ College of Mathematics and Information Science \\ Henan Normal University \\ Xinxiang 453007, China \\ e-mail: mayingbincw@htu.cn
}

\begin{abstract}
A total-colored path is total rainbow if both its edges and internal vertices have distinct colors. The total rainbow connection number of a connected graph $G$, denoted by $\operatorname{trc}(G)$, is the smallest number of colors that are needed in a total-coloring of $G$ in order to make $G$ total rainbow connected, that is, any two vertices of $G$ are connected by a total rainbow path. In this paper, we study the computational complexity of total rainbow connection of graphs. We show that deciding whether a given total-coloring of a graph $G$ makes it total rainbow connected is NP-Complete. We also prove that given a graph $G$, deciding whether $\operatorname{trc}(G)=3$ is NP-Complete.
\end{abstract}

Keywords: total rainbow connection, computational complexity.

2010 Mathematics Subject Classification: 05C15, 05C40, 68Q25, 68R10. 


\section{REFERENCES}

[1] J.A. Bondy and U.S.R. Murty, Graph Theory (GTM 244, Springer, 2008).

[2] Y. Caro, A. Lev, Y. Roditty, Zs. Tuza and R. Yuster, On rainbow connection, Electron J. Combin. 15 (2008) R57.

[3] S. Chakraborty, E. Fischer, A. Matsliah and R. Yuster, Hardness and algorithms for rainbow connectivity, J. Comb. Optim. 21 (2011) 330-347. doi:10.1007/s10878-009-9250-9

[4] G. Chartrand, G.L. Johns, K.A. McKeon and P. Zhang, Rainbow connection in graphs, Math. Bohem. 133 (2008) 85-98.

[5] L. Chen, X. Li and Y. Shi, The complexity of determining the rainbow vertexconnection of graphs, Theoret. Comput. Sci. 412 (2011) 4531-4535. doi:10.1016/j.tcs.2011.04.032

[6] M. Garey, D.S. Johnson and L.J. Stockmeyer, Some simplified NP-complete graph problems, Theoret. Comput. Sci. 1 (1976) 237-267. doi:10.1016/0304-3975(76)90059-1

[7] X. Huang, X. Li and Y. Shi, Note on the hardness of rainbow connections for planar and line graphs, Bull. Malays. Math. Sci. Soc. 88 (2015) 1235-1241. doi:10.1007/s40840-014-0077-x

[8] X. Huang, X. Li, Y. Shi, J. Yue and Y. Zhao, Rainbow connections for outerplanar graphs with diameter 2 and 3, Appl. Math. Comput. 242 (2014) 277-280. doi:10.1016/j.amc.2014.05.066

[9] M. Krivelevich and R. Yuster, The rainbow connection of a graph is (at most) reciprocal to its minimum degree, J. Graph Theory 63 (2010) 185-191.

[10] S. Li, X. Li and Y. Shi, Note on the complexity of deciding the rainbow (vertex-) connectedness for bipartite graphs, Appl. Math. Comput. 258 (2015) 155-161. doi:10.1016/j.amc.2015.02.015

[11] X. Li, Y. Mao and Y. Shi, The strong rainbow vertex-connection of graphs, Util. Math. 93 (2014) 213-223.

[12] X. Li and Y. Shi, On the rainbow vertex-connection, Discuss. Math. Graph Theory 33 (2013) 307-313. doi:10.7151/dmgt.1664

[13] X. Li and Y. Shi, Rainbow connection in 3-connected graphs, Graphs Combin. 29 (2013) 1471-1475. doi:10.1007/s00373-012-1204-9

[14] X. Li, Y. Shi and Y. Sun, Rainbow connections of graphs: A survey, Graphs Combin. 29 (2013) 1-38. doi:10.1007/s00373-012-1243-2

[15] X. Li and Y. Sun, Rainbow Connections of Graphs (New York, Springer Briefs in Math., Springer, 2012). 
[16] H. Liu, A. Mestre and T. Sousa, Total rainbow k-connection in graphs, Discrete Appl. Math. 174 (2014) 92-101. doi:10.1016/j.dam.2014.04.012

[17] I. Schiermeyer, Rainbow connection in graphs with minimum degree three, IWOCA 2009, Lecture Notes in Comput. Sci. 5874 (2009) 432-437.

[18] K. Uchizawa, T. Aoki, T. Ito, A. Suzuki and X. Zhou, On the rainbow connectivity of graphs: Complexity and FPT algorithms, Algorithmica 67 (2013) 161-179. doi:10.1007/s00453-012-9689-4

Received 10 March 2015

Revised 7 July 2015

Accepted 7 July 2015 